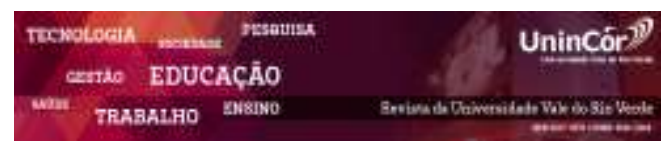

Revista da Universidade Vale do Rio Verde ISSN: 1517-0276 / EISSN: 2236-5362 Vol. 16 | n. 2 | Ano 2018

Bianca dos Santos Costa Universidade do Estado de Santa Catarina biancasantoscosta0111@gmail.com

Jaqueline Gonçalves Universidade do Estado de Santa Catarina jaquelinegoncalves-@ hotmail.com

Fabiano Maury Raupp Universidade do Estado de Santa Catarina fabianoraupp@hotmail.com

\section{POSSIBILIDADES E PERSPECTIVAS DA APROPRIAÇÃO DE CUSTOS NO AUMENTO DA EFICIÊNCIA DO SETOR PÚBLICO}

\begin{abstract}
RESUMO
O presente artigo discute a importância do conhecimento do custo dos serviços e bens públicos no contexto da necessidade de informações amplas e confiáveis para o controle das contas públicas. Para atingir o objetivo proposto foi desenvolvida uma discussão com base em artigos científicos que analisaram a percepção de gestores sobre a utilização de sistema de custos públicos, identificando as principais mudanças, dificuldades e perspectivas observadas no processo de implantação. Por intermédio de pesquisa em bases de dados, obteve-se a seleção de 44 artigos, o qual resultou em 07 artigos relevantes de acordo com os critérios que foram definidos na pesquisa. Através da análise e discussão realizadas foi possível identificar aspectos qualitativos para demonstrar o aumento da eficiência no setor público através do controle dos custos.
\end{abstract}

Palavras-chave: Custos. Eficiência. Setor Público.

\begin{abstract}
This article discusses the importance of knowledge of the cost of services and public goods in the context of the need for large and reliable information to control public accounts. In order to achieve the proposed goal, a discussion was developed based on scientific articles that analyzed the managers' perceptions about the use of public cost system, identifying the main changes, difficulties and perspectives observed in the implementation process. Through a database search, the selection of 44 articles was obtained, which resulted in 07 relevant articles according to the criteria that were defined in the research. Through the analysis and discussion it was possible to identify qualitative aspects to demonstrate the increase of efficiency in the public sector through cost control.
\end{abstract}

Keywords: Costs. Efficiency. Public Sector.

Recebido em: 10/01/2018 - Aprovado em: 25/07/2018 - Disponibilizado em: 15/12/2018 


\section{INTRODUÇÂO}

A sociedade encontra-se cada vez mais exigente com relação ao acompanhamento e supervisão dos gastos públicos, cobrando por ações mais transparentes e rentáveis para a administração pública. A implantação de sistema de custos pelas entidades governamentais representa uma ferramenta que pode auxiliar no reconhecimento da eficiência e economia das organizações, proporcionando uma série de melhorias tanto para o cidadão quanto para os próprios gestores. Conforme comenta Alonso (1999), a realização correta da apuração dos custos dos serviços e da publicidade dos mesmos representam dois importantes instrumentos de controle social que proporciona aos auditores e usuários uma avaliação eficiente dos serviços prestados.

Tendo em vista que a gestão de custos públicos no Brasil é um tema em fase de desenvolvimento e que a grande maioria das organizações públicas ainda se encontra em processo de implantação de sistema de informação de custos, surge a questão-problema que norteia este estudo: Quais as possibilidades e perspectivas que podem ser observadas na administração pública com o aumento do controle dos custos?

Com o intuito de responder à pergunta de pesquisa, busca-se como objetivo analisar a relevância da adoção de um sistema de custos para um aumento da eficiência no setor público. Nesse contexto, a justificativa encontrada para a realização desta pesquisa está na demonstração de experiências com relação à utilização de sistema de custos públicos no Brasil, bem como informar sobre os pontos críticos apontados por quem já utiliza esse tipo de sistema de gerenciamento de custos.

A importância do estudo pode ser confirmada pela obrigatoriedade da implantação do sistema de custos em todas as entidades públicas, porquanto se trata de um tema que todos os entes da federação já deveriam estar implementado, devido o prazo para a obrigatoriedade estabelecido pelas regras da Portaria STN $n^{\circ}$ 406/2011, determinando que cada entidade estabeleça um cronograma de ações que possibilitasse dar conhecimento do sistema de custos aos gestores e possíveis interessados, motivo pelo qual esta pesquisa busca demonstrar a importância da adoção de um sistema de custos para um aumento da eficiência no setor público.

\section{REFERENCIAL TEÓRICO}

\subsection{Sistema de Custos no Setor Público}

O Sistema de Informação de Custos do Setor Público - SICSP foi instituído pelas normas brasileiras de contabilidade aplicada ao setor público, na qual criou a obrigatoriedade de implantação para todas as entidades governamentais. Este sistema é ainda muito recente se comparado às empresas privadas, visto que sua obrigatoriedade foi instituída pela NBC T 16.2 - Patrimônio e sistemas contábeis, aprovada pela resolução do CFC $\mathrm{n}^{\circ}$. 1.129/2008, na qual apresentou as alterações nos sistemas de informação contábeis utilizados no Brasil. Carneiro et al. (2013) afirma que até 2009 a 
contabilidade pública se dividia em quatro sistemas, sendo eles: Orçamentário, Financeiro, Patrimonial e Compensação, tendo sido acrescentado o sistema "Custos" apenas após a aprovação da NBC T 16.2.

Entretanto, a temática relacionada à criação de uma contabilidade especial para apuração dos custos nos serviços públicos já vem sendo disposta desde a Lei Federal no 4.320, de 17 de março de 1964, na qual a menção aos custos é feita no art. 85 e art. 99, apresentando a necessidade da determinação dos custos para os serviços industriais. A partir da publicação do Decreto Lei $n^{\circ} 200$, de 25 de fevereiro de 1967, a temática dos custos no setor público apresentou uma finalidade mais gerencial, evidenciando em seu art. 79 que os custos dos serviços deveriam ser apurados pela contabilidade a fim de apresentar os resultados da gestão.

A Lei de Responsabilidade Fiscal de $n^{\circ}$ 101, de 04 de maio de 2000, também reforçou a obrigatoriedade da utilização de informações de custos para os serviços públicos, destacando em seu art. 50, $\S 3^{\circ}$ que toda a Administração Pública deveria manter um sistema de custos que permita o acompanhamento e a avaliação da gestão orçamentária, financeira e patrimonial.

O Conselho Federal de Contabilidade publicou a norma NBC T 16.11, específica sobre Sistema de Informação de Custos do Setor Público - SICSP, aprovada pela resolução CFC $n^{\circ} 1.366 / 2011$, na qual estabelece os conceitos, objetivos e regras para evidenciação e mensuração dos custos no setor público. Conforme evidenciado nesta norma, os custos dos bens e serviços e outros objetos de custos que forem produzidos e oferecidos à sociedade pela entidade pública, deverão ser registrados, processados e evidenciados pelo SICSP. (CFC, 2011).

Apesar de a exigência legal de utilização de sistema de informação de custos no setor público já ter sido contemplada desde o ano 1964, a obrigatoriedade de implementação só ficou estabelecida quando a Secretaria do Tesouro Nacional publicou a Portaria STN n ${ }^{\circ}$ 406, de 20 de junho de 2011, que posteriormente foi alterada pela Portaria STN n ${ }^{\circ} 828 / 2011$, de 14 de dezembro de 2011 e pela Portaria STN n ${ }^{\circ}$ 231, de 29 de março de 2012, determinando que a União, Estados/DF e Municípios se adequassem aos novos dispositivos legais vigentes, estabelecendo prazos para a implementação dos procedimentos contábeis patrimoniais e determinando que cada organização pública realizasse a elaboração de um cronograma de ações que deveria ser adotado até o final do exercício de 2014. Entretanto, as experiências em custos no setor público são ainda muito pequenas, conforme verificados nos estudos de Costa, Dias e Raupp (2017) nenhum dos Estados brasileiros conseguiu efetivamente implementar esse sistema de custos em seus governos até o ano de 2017.

\subsection{Eficiência no Setor Público}

O conhecimento dos custos dos serviços prestados à sociedade pode contribuir tanto para o gestor na tomada de decisão, como para os próprios usuários do serviço público na verificação da gestão eficiente dos recursos, possibilitando maior transparência e controle social. Conforme declara Costa, Dias e Raupp 
(2017), a sociedade está cada vez mais preocupada com a maneira como são realizados os gastos públicos, cobrando por melhorias na qualidade dos serviços, bem como exigindo maior transparência nas ações do governo.

Segundo Slomski (2010), podemos observar a eficiência na prestação de serviços de uma entidade, pela demonstração dos resultados econômicos, quando estes são alinhados com os sistemas de custos. Demonstrando que a organização e a integração dos custos das organizações se mostram eficientes ao ponto de representar importantes indicadores de desempenho.

O sistema de informação de custos representa uma ferramenta que poderá auxiliar na formulação e na organização dos dados gerados pelas organizações, possibilitando da mesma forma uma adequação às legislações de custos no setor público. Segundo Machado (2002, p. 146) o objetivo de um sistema de custos "é prover informações que auxiliem a tomada de decisão do gestor público, bem como o controle e avaliação da eficiência, eficácia e da efetividade, relativos aos programas em execução ou já executados".

O princípio da eficiência foi introduzido ao art. 37 da Constituição do Brasil no ano de 1998, por meio da Emenda Constitucional $\mathrm{n}^{\circ}$ 19/1998, na qual a nova redação deste artigo determinou o seguinte: “A administração pública direta e indireta de qualquer dos poderes da União, dos Estados, do Distrito Federal e dos Municípios obedecerá aos princípios de legalidade, impessoalidade, moralidade, publicidade e eficiência." Logo, a eficiência passou a ser um dos princípios da administração pública, proporcionando o surgimento de novas políticas que auxiliem essa questão, como é o caso do surgimento dos sistemas de custos no setor público.

\section{PROCEDIMENTOS METODOLÓGICOS}

Os procedimentos adotados no estudo consideraram o perfil científico do estudo, evidenciando e classificando os meios utilizados para a obtenção das informações que sustentam a pesquisa. Com o intuito de atingir o objetivo desta pesquisa, desenvolveu-se um estudo de natureza descritiva, uma vez que busca descrever a percepção de alguns gestores públicos a respeito da implantação do sistema de custos, a partir de uma busca em artigos publicados sobre o tema. Segundo Andrade (2010), a pesquisa descritiva é aquela em que os fatos são observados, registrados e analisados de forma que o pesquisador não interfira nas informações geradas.

Esta pesquisa também pode ser classificada como bibliográfica, posto que possui a finalidade de fazer uma seleção em artigos publicados no Portal de Periódicos da CAPES sobre os sistemas de custos no setor público, buscando fazer uma análise sobre os artigos encontrados a respeito da percepção dos gestores públicos envolvidos no processo de implantação. Severino (2016) esclarece que este tipo de pesquisa é realizado a partir do registro disponível, decorrente de pesquisas anteriores, utilizando dados já trabalhados por outros pesquisadores e corretamente registrados. 
Em relação à abordagem do problema, a pesquisa caracterizou-se como qualitativa, pois tem como foco principal descrever as principais dificuldades e contribuições advindas da apropriação dos custos no setor público.

Por intermédio deste procedimento foram coletados dados primários que foram utilizados no referencial teórico e dados secundários predominantemente para seleção dos artigos na base de dados da CAPES. A escolha dos artigos foi realizada por meio de consulta no portal de periódicos da CAPES, utilizando a expressão booleana AND e OR e não restringir a pesquisa simultânea no título, resumo e palavraschave dos artigos, para o qual foram definidas as seguintes palavras-chave: "Sistema de Custos" OR "Contabilidade de Custos" AND "Setor Público".

De acordo com estes critérios, a coleta de dados resultou num total de 44 artigos. De posse destes artigos, fez se primeiramente a leitura do resumo, que serviu de base para seleção de 07 artigos que tinham como objetivo apresentar a percepção dos gestores sobre a implantação de um sistema de custo. O motivo da escolha desses artigos deu-se principalmente pela análise realizada por esses estudos, apresentando os fatores e as perspectivas de gestores a respeito do aumento da eficiência do setor público através da apropriação dos custos. Dos artigos desclassificados 08 estavam repetidos, 04 apresentam estudos embasados na teoria, 08 apresentam estudos relacionados à saúde, 02 apresentam estudos relacionados à educação e 15 faziam uma análise mais diferenciada sobre custos.

\section{RESULTADOS DA PESQUISA}

\subsection{Experiências em Curso (casos)}

O trabalho de Carneiro et al. (2013), abordou os principais desafios, obstáculos e percepção dos servidores e gestores do município de Vilhena - Rondônia, a respeito dos principais fatores que determinam as dificuldades para implantação do sistema de custos no setor público municipal. Os autores realizaram um estudo de caso em que os dados foram obtidos a partir de 17 respostas recebidas. Os resultados apontaram que as principais dificuldades identificadas na pesquisa foram à falta de conhecimento do gestor, a falta de interesse desse tipo de controle, a falta de servidores especializados, falta de estudos na área e de planejamento das atividades.

Silva e Rosa (2015), verificaram a percepção de contadores de prefeituras municipais do Estado de Santa Catarina, a respeito da implantação de um sistema de custos para a administração pública. A coleta dos dados foi obtida por meio de questionário enviado aos contadores de prefeituras municipais, no qual se obteve respostas de $32,88 \%$ da população. Os resultados apresentaram que os contadores municipais reconhecem a importância da implantação de um sistema de custos para a administração pública, sendo que apenas 01 prefeitura efetivamente implantou um sistema de contabilidade de custos e outras 14 estavam em processo de implantação.

O estudo de Pereira e Araujo (2016) teve como objetivo analisar a percepção dos Auditores e Analistas das Inspetorias de 
Contabilidade e Finanças do Exército (ICFEx) sobre o Sistema de Custos Gerencial (SISCUSTOS), analisando se este gera informações relevantes sobre a apuração de custos. Foi realizado um estudo de caso com amostra formada por 88 profissionais de contabilidade que analisam o sistema do Exército Brasileiro. Para os Analistas e Auditores das ICFEx, 45,83\% concordam totalmente que a legislação alterou a forma de trabalho em relação às informações geradas. Sendo possível considerar que, na percepção dos Analistas e Auditores das ICFEx, existe o uso da contabilidade de custos, e que o SISCUSTOS está adequado as normas de contabilidade pública.

Almeida, Borba e Flores (2009), analisaram o uso das informações de custos pelos gestores de 20 grandes secretarias municipais da saúde do estado de Santa Catarina. Avaliando o perfil dos gestores entrevistados; verificando a existência ou não de sistemas de custos implantados nessas secretarias; fazendo uma avaliação da percepção desses gestores sobre a utilização das informações de custos no processo de tomada de decisão e foram identificadas quais informações de custos são utilizadas atualmente na gestão desses órgãos. Conseguiu-se avaliar o uso das informações de custos na gestão de 18 secretarias municipais da saúde do estado de Santa Catarina. Os resultados indicam que apenas duas secretarias municipais da saúde possuem esse processo um pouco mais avançado; nas demais, o que se verifica é que existem algumas tentativas de se obter informações mais detalhadas sobre custos. Entretanto, na opinião dos entrevistados, as informações de custos são bastante importantes na gestão das secretarias.

Pereira e Romão (2015) apresentam como objetivos principais avaliar a implementação do Sistema de Contabilidade de Custos nos municípios portugueses, bem como analisar a utilidade da informação produzida por este sistema. Os autores utilizam um questionário distribuído pelos 308 municípios como ferramenta da investigação. Os resultados demonstraram que apenas $58 \%$ dos inquiridos já utilizam o SICSP numa fase de implementação intermédia.

Franco et al. (2013) apresentam um estudo que tem como objetivo conhecer o nível de entendimento e de adoção quanto aos sistemas de custos por parte dos municípios do Paraná. Sendo realizada a aplicação de um questionário em 67 municípios do Estado do Paraná para coleta de dados. Dentre os resultados do estudo, destacam-se: a incipiência do conhecimento acerca da importância, viabilidade e utilidade de um sistema de custos; embasado por limitações no conhecimento por parte dos agentes públicos, sobretudo, nos tópicos que tratam de viabilidade orçamentária e financeira da adoção de sistemas de custos; ambiente para o sistema de custos, sistemas de custos implantados e sistemas operacionais;

Machado e Holanda (2010) analisaram o Sistema de Informação de Custos do Governo Federal, descrevendo o modelo e suas principais características, desde o conceito até a finalidade e os grupos de usuários, explorando a correspondência entre os conceitos de contabilidade governamental e de custos. O artigo demonstra o relacionamento entre as 
políticas públicas, recursos, atividades e objetos de custo.

Diante dos estudos apresentados, percebe-se que as pesquisas sobre os sistemas de informação de custos no Brasil continuam escassas, mas que há uma tendência em se expandir análises nessa área, principalmente pela importância dada ao conhecimento dos custos públicos para gerenciamento das organizações públicas.

\subsection{Análises das Dificuldades}

$\mathrm{Na}$ análise realizada, verificou-se que dos estudos pesquisados apenas os artigos de Almeida, Borba e Flores (2009), Silva e Rosa (2015) e Pereira e Romão (2015) apresentam casos onde já foi efetivamente implementado um sistema de contabilidade de custos, os demais estão em processo inicial de implantação, ou apresentam apenas a intenção em aderir esse tipo de sistema.

Por meio do Quadro 1 pode-se observar as principais dificuldades relacionadas com a implementação do sistema de custos, apresentados em cada artigo, segundo a perspectiva dos respondentes: funcionários e gestores públicos.

Quadro 1 - Análise das dificuldades

\begin{tabular}{|c|c|c|c|c|c|c|c|}
\hline Dificuldades & $\begin{array}{c}\text { Pereira e } \\
\text { Romão } \\
(2015)\end{array}$ & $\begin{array}{c}\text { Franco } \\
\text { et al } \\
(2013)\end{array}$ & $\begin{array}{c}\text { Pereira e } \\
\text { Araujo } \\
(2016)\end{array}$ & $\begin{array}{c}\text { Silva e } \\
\text { Rosa } \\
(2015)\end{array}$ & $\begin{array}{c}\text { Machado e } \\
\text { Holanda } \\
(2010)\end{array}$ & $\begin{array}{c}\text { Correia } \\
\text { et al } \\
(2012)\end{array}$ & $\begin{array}{c}\text { Almeida, } \\
\text { Borba e } \\
\text { Flores } \\
(2009)\end{array}$ \\
\hline $\begin{array}{l}\text { Falta capacitação de servidores / } \\
\text { Escassez de recursos humanos }\end{array}$ & $X$ & $\mathrm{X}$ & & $\mathrm{X}$ & & $X$ & \\
\hline $\begin{array}{l}\text { Falta de um modelo para } \\
\text { implementação }\end{array}$ & $\mathrm{X}$ & & $X$ & $X$ & $X$ & $X$ & \\
\hline $\begin{array}{l}\text { Falta de recursos orçamentários e } \\
\text { financeiros }\end{array}$ & $X$ & $\mathrm{X}$ & & & & $X$ & $\mathrm{X}$ \\
\hline $\begin{array}{l}\text { Resistência por parte dos } \\
\text { funcionários e/ou gestores públicos }\end{array}$ & $\mathrm{X}$ & $X$ & $X$ & & & $X$ & \\
\hline $\begin{array}{l}\text { Falta de compartilhamentos de } \\
\text { dados entre as áreas }\end{array}$ & & $X$ & $X$ & & $X$ & & $\mathrm{X}$ \\
\hline Falta de planejamento & & $\mathrm{X}$ & & $\mathrm{X}$ & & $\mathrm{X}$ & \\
\hline Falta de uma Legislação & & & $\mathrm{X}$ & & & $\mathrm{X}$ & $\mathrm{X}$ \\
\hline Escassez de Meios Técnicos & $\mathrm{X}$ & $\mathrm{X}$ & & & & & \\
\hline $\begin{array}{l}\text { Falta de Mecanismos de } \\
\text { Fiscalização }\end{array}$ & $\mathrm{X}$ & & & & & & $\mathrm{X}$ \\
\hline
\end{tabular}

Fonte: Dados da pesquisa (2017)

Foi possível identificar nesses estudos as principais dificuldades relacionadas à implantação do sistema de custos, visto que foram encontrados basicamente os mesmos problemas em cada artigo. Destaca-se que a falta de um modelo para a implementação do sistema de custos é um dos fatores mais recorrentes, posto que foi citado em 05 dos 07 artigos 
selecionados. A maior reclamação sobre esse tema persiste na falta de informação por parte dos servidores, que na ausência de um modelo base precisam se adaptar à realidade de cada órgão.

Outro fator de grande dificuldade identificado está relacionado com a quantidade de integrantes responsáveis por este processo de implantação de sistema de custos em cada organização. Destacando que não é apenas a falta de pessoal o problema, mas sim a falta de pessoal capacitado que possua conhecimento das atividades de custos. Foram evidenciados nesses artigos que muitos dos respondentes não possuíam informações suficientes sobre a portaria de implantação ou sobre os próprios sistemas.

No artigo de Silva e Rosa (2015), fica demonstrado com dados estatísticos que os municípios que já haviam implementado ou estavam em processo de implantação, possuíam profissionais com maior informação sobre o tema que os municípios que ainda não iniciaram a utilização desse sistema. Sendo necessário que cada instituição pública invista em mais treinamentos e capacitações. Além disso, alguns dos artigos demonstra também que os profissionais responsáveis por esse processo de implantação de sistema de custos nas instituições normalmente não possuem acesso a maior parte das informações necessárias.

A falta de compreensão dos funcionários públicos sobre a real utilidade da implantação dos sistemas de custos também representou uma reclamação persistente entre os estudos analisados. Muitos dos servidores entrevistados nos artigos selecionados, apresentaram ressalvas quanto à demanda de trabalho envolvida para a implementação efetiva deste sistema, além da ausência de planejamento e de cronogramas que tornam a implementação do sistema de custos mais dispendiosa e prolongada. Sem punições preestabelecidas muitos sistemas de custos acabam tendo seu processo de implantação adiados, sendo que a prioridade é dada a atividades que apresentem prazos e multas.

Em resumo, destaca-se as principais dificuldades encontradas nos artigos selecionados: escassez de recursos humanos capacitados; escassez de modelos para implementação; escassez de recursos financeiros e orçamentários; resistência por parte dos funcionários e gestores; falta de compartilhamento de informações; falta de fiscalização; falta de planejamento; falta de informações detalhadas sobre a portaria; escassez dos meios técnicos, como sistemas;

Uma das dificuldades pouco comentada, mas que acarreta grande impacto no processo de implantação é a alta rotatividade na gestão, bem como da equipe de custos. Acredita-se que se deve ao fato de haver muitas questões políticas envolvidas, visto que grande parte do trabalho desenvolvido ao longo do mandato acaba sendo perdido na nova gestão.

Os artigos de Carneiro et al. (2013) e Pereira e Romão (2015), apresentam que após as instituições já possuírem sistema de custos implantado, também existe a dificuldade dos gestores e usuários em utilizar as informações apresentadas pelos próprios sistemas. Mostrando que se o acompanhamento dos custos não for feito de forma completa as informações adquiridas serão mal interpretadas. Machado e 
Holanda (2010, p. 818) apresentam similar leitura, mostrando que a utilização das informações adquiridas pelo sistema de custos é baixa. "A par de todas as possibilidades, o maior desafio é fazer com que as informações geradas sejam úteis e apropriadas ao processo decisório".

\subsection{Análise das Perspectivas}

Neste tópico buscou-se apresentar as principais contribuições e perspectivas relacionadas ao processo de implantação de sistema de custos pelos gestores públicos analisados dos artigos selecionados, o Quadro 2 demonstra os resultados obtidos.

Quadro 2 - Contribuições

\begin{tabular}{|l|c|c|c|c|c|c|c|}
\hline \multicolumn{1}{|c|}{ Perspectivas } & $\begin{array}{c}\text { Pereira e } \\
\text { Romão } \\
(2015)\end{array}$ & $\begin{array}{c}\text { Franco } \\
\text { et al } \\
(2013)\end{array}$ & $\begin{array}{c}\text { Pereira e } \\
\text { Araujo } \\
(2016)\end{array}$ & $\begin{array}{c}\text { Silva e } \\
\text { Rosa } \\
(2015)\end{array}$ & $\begin{array}{c}\text { Machado e } \\
\text { Holanda } \\
(2010)\end{array}$ & $\begin{array}{c}\text { Correia } \\
\text { et al } \\
(2012)\end{array}$ & $\begin{array}{c}\text { Almeida, } \\
\text { Borba e } \\
\text { Flores } \\
(2009)\end{array}$ \\
\hline Maior transparência nas Finanças & $\mathrm{X}$ & & & $\mathrm{X}$ & & $\mathrm{X}$ & \\
\hline $\begin{array}{l}\text { Facilita a avaliação de desempenho } \\
\text { dos Órgãos Públicos }\end{array}$ & & $\mathrm{X}$ & $\mathrm{X}$ & & $\mathrm{X}$ & $\mathrm{X}$ \\
\hline Maior controle sobre os gastos & $\mathrm{X}$ & & $\mathrm{X}$ & $\mathrm{X}$ & & $\mathrm{X}$ \\
\hline $\begin{array}{l}\text { Aumento da economia, eficiência e } \\
\text { eficácia }\end{array}$ & $\mathrm{X}$ & & $\mathrm{X}$ & & & $\mathrm{X}$ \\
\hline $\begin{array}{l}\text { Suporte ao processo de tomada de } \\
\text { decisão }\end{array}$ & $\mathrm{X}$ & & $\mathrm{X}$ & $\mathrm{X}$ & $\mathrm{X}$ & $\mathrm{X}$ & $\mathrm{X}$ \\
\hline Melhoria da gestão & $\mathrm{X}$ & & $\mathrm{X}$ & $\mathrm{X}$ & & $\mathrm{X}$ & \\
\hline
\end{tabular}

Fonte: Dados da pesquisa (2017)

Por meio das assertivas evidenciadas no quadro acima, constata-se que houve um domínio na concordância das contribuições, demonstrando que os gestores envolvidos no processo de implantação do sistema de custos nesses artigos acreditam nas potenciais vantagens e benefícios que podem ser obtidos da utilização efetiva dessa ferramenta de gestão.

O conhecimento mais detalhado dos custos torna mais simples a identificação e análise de serviços, tornando a compra mais segura e com menos desperdícios financeiros. Além disso é possível também fazer análises mais avançadas, como perceber a necessidade de cancelamento e ou melhoria dos mesmos, sendo este um fator apontado na pesquisa de Pereira e Araújo (2016).
$\mathrm{Na}$ pesquisa Carneiro et al. (2013), a maior vantagem apresentada pela implementação do sistema de custos é o controle financeiro e o auxílio para a tomada de decisão, mostrando-se assim, uma excelente ferramenta de gestão. Já para Silva e Rosa (2015) a transparência representa um dos fatores que permitem alcançar um dos mais vantajosos resultados na implantação deste sistema de custo.

Em resumo, destacamos as principais melhorias relacionadas com a implantação de sistema de custos, segundo os artigos selecionados: auxílio na tomada de decisão; aumento do controle de gastos; aumento da economia eficácia e eficiência; melhoria dos serviços públicos; aumento da transparência; aumento do desempenho dos órgãos públicos. 
A utilização dos dados e informações de custos como ferramenta para a tomada de decisão está entre as vantagens mais citadas entre os artigos. Além de ser uma ótima ferramenta financeira, as informações geradas pelo sistema de custos permitem tomar decisões importantes para os órgãos, como quanto à permanência ou exclusão de determinados serviços, a realocação de recursos, corte de gastos. Outro fator comentado em diversos dos artigos é sobre a utilidade do sistema como fator de avaliação do desempenho dos órgãos públicos.

\section{CONCLUSÃO}

Esta pesquisa apresentou como objetivo relatar a relevância da adoção de um sistema de custos para um aumento da eficiência no setor público, a partir da análise de estudos publicados no portal de periódicos da CAPES que tivessem pesquisas com foco na temática de custos aplicados ao setor público, mediante um estudo comparativo entre as mudanças que podem ser verificadas após a utilização de uma ferramenta de gestão de custos, destacando as principais dificuldades e perspectivas que podem ser observadas em um processo de implantação de sistema de custos. Para tanto, adotou-se uma pesquisa descritiva, qualitativa e bibliográfica.

Embora ainda seja muito baixo o número de instituições públicas que implementaram efetivamente um sistema de informação de custos, é possível constatar que em todos os artigos selecionados para esta pesquisa, o processo de implantação só começou a ser estudado após a emissão da Portaria STN $\mathrm{n}^{\circ} 406 / 2011$, que estabeleceu prazos para a obrigatoriedade de implantação de um sistema de custos para todas as esferas do setor público.

Os resultados da pesquisa demonstram que as principais dificuldades identificadas pelos respondentes dos artigos selecionados referente ao processo de implantação de sistema de custos são a falta de padronização dos modelos de sistema de custos, escassez de profissionais capacitados, falta de recursos financeiros e orçamentários, falta de fiscalização e meios técnicos. Entretanto, acredita-se que deva existir outras dificuldades que possam impedir a implantação de sistema de custos, visto que muitas entidades avaliadas nos artigos não possuem ainda sistema de custos implantado.

$$
\text { Com relação as perspectivas }
$$
relacionadas ao processo de implantação de sistema de custos foram identificadas como contribuições um maior auxílio na tomada de decisão, maior controle dos gastos, aumento da economia, eficácia e eficiência, bem como melhorias dos serviços públicos por meio do aumento da transparência.

Por fim, pode-se concluir que o sistema de custos não representa apenas uma ferramenta de controle financeiro e orçamentário, posto que suas informações geram impactos e melhorias dentro de todos os setores da organização. A utilização das informações do sistema de custo além de permitir um maior controle dos gastos públicos, geram uma maior eficiência no desempenho das instituições governamentais.

Como sugestão, recomenda-se a aplicação de uma pesquisa mais ampla, que contemple um maior número de artigos, 
publicados em outros portais, congressos ou revistas, a fim de verificar a questão da eficiência e da padronização nas três esferas do governo.

\section{REFERÊNCIAS}

ALMEIDA, A. G. de; BORBA, José A.; FLORES, L. C. S. A utilização das informações de custos na gestão da saúde pública: um estudo preliminar em secretarias municipais de saúde do estado de Santa Catarina. Revista de

Administração Pública, Rio de Janeiro, v. 43, p. 579-607, 2009.

ALONSO, M. Custos no serviço público.

Revista do Serviço Público, v. 50, n. 1, p. 37 63, 1999.

ANDRADE, M. M. de. Introdução a metodologia do trabalho científico: elaboração de trabalhos na graduação. São Paulo: Atlas, 2010.

BRASIL. Decreto-Lei n ${ }^{\circ} 200$, de 25 de fevereiro de 1967. Dispõe sobre a organização da administração federal, estabelece diretrizes para a Reforma Administrativa e da outras providências. Disponível em: $<$ http://www.planalto.gov.br/ccivil_03/decretolei/Del0200.htm>. Acesso em: 20/11/2017.

\section{Constituição da República Federativa}

do Brasil. Brasília: Presidência da República, 1988.

Emenda Constitucional no 19, de 04 de junho de 1998 - Modifica o regime e dispõe sobre princípios e normas da Administração Pública, servidores e agentes políticos, controle de despesas e finanças públicas e custeio de atividades a cargo do Distrito Federal, e dá outras providências. Disponível em: <http://www.planalto.gov.br/ccivil_03/constituic ao/emendas/emc/emc19.htm>. Acesso em: 20/11/2017.
Lei Complementar no 101, de 04 de maio de 2000. Estabelece normas de finanças públicas voltadas para a responsabilidade na gestão fiscal e dá outras providências.

Disponível em:

<http://www.planalto.gov.br/ccivil_03/leis/LCP/ Lcp101.htm>. Acesso em: 20/11/2017.

Lei $\mathbf{n}^{\mathbf{0}}$ 4.320, de 17 de março de 1964 Estatui normas gerais de direito financeiro para elaboração e controle dos orçamentos e balanços da União, Estados, Municípios e do Distrito Federal. Disponível em: <http://www.planalto.gov.br/ccivil_03/leis/L432 0.htm>. Acesso em: 20/11/2017.

CARNEIRO, A. de F. et al. Sistema de custos municipal: desafios, obstáculos e percepção no caso do município de Vilhena, Rondônia.

Revista Ambiente Contábil, v. 5, n. 2, p. 53-67, 2013.

\section{CONSELHO FEDERAL DE}

\section{CONTABILIDADE - CFC. Resolução n. ${ }^{\circ}$}

1.129, de 21 de novembro de 2008: aprova a NBC T 16.2 - patrimônio e sistemas contábeis, 2008. Disponível em:

http://www.cfc.org.br/sisweb/sre/detalhes_sre.as px ? Codigo $=2008 / 001129$. Acesso em: 27/10/2017.

Resolução n. ${ }^{0} \mathbf{1 . 3 6 6}$, de 25 de novembro de 2011: aprova a NBC T 16.11 Sistema de Informação de Custos do Setor Público. 2011. Disponível em: <http://www.cfc.org.br/sisweb/sre/detalhes_sre.a spx ?Codigo=2011/001366>. Acesso em: 27/10/2017.

COSTA, B. dos S.; DIAS, J. da Silva, RAUPP, F. M. Sistema de custos no setor público: um estudo nos Estados brasileiros face a realidade de implantação. In: Anais do Congresso Brasileiro de Custos-ABC. 2017. Disponível em: <https://anaiscbc.emnuvens.com.br/anais/article/ view/4327/4327>. Acesso em: 05/12/2017.

SILVA, L. C. de; ROSA, F. S. da. Aspectos de convergência da contabilidade no setor público: um enfoque sobre a implantação do 
sistema de custos segundo a percepção de contadores municipais do Estado de Santa Catarina. Contabilidad y Negocios: Revista del Departamento Académico de Ciencias Administrativas, 2015, Vol.10. Disponível em: $<$ http://revistas.pucp.edu.pe/index.php/contabilid adyNegocios/article/view/14086/14856>. Acesso em: 05/12/2017.

FRANCO, L. M. G. et al. Sistemas de custos: importância, viabilidade e utilidade na concepção dos agentes públicos do Estado do Paraná (Brasil). Revista de Educação e Pesquisa em Contabilidade, v. 7, n. 2, p. 167183, 2013.

\section{MACHADO, Nelson. Sistema de informação de custo: diretrizes para integração ao orçamento público e à contabilidade governamental. 2002. 221p. Tese (Doutorado em Controladoria e Contabilidade) - Faculdade de Economia, Administração e Contabilidade, Universidade São Paulo, São Paulo.}

MACHADO, Nelson; HOLANDA, Victor B. Diretrizes e modelo conceitual de custos para o setor público a partir da experiência no governo federal do Brasil. Revista de Administração Pública, v. 44, n. 4, p. 791-820, 2010.

PEREIRA, T. R. L.; ARAUJO, A. O. Relevância das informações geradas pelo Siscustos para a nova administração pública, na percepção dos analistas e auditores das ICFEx. Revista

Contemporânea de Contabilidade, v. 13, n. 28, p.169-189, 2016.

PEREIRA, A. L.; ROMÃO, A. L. A implementação do sistema de contabilidade de custos nos municípios portugueses. Revista

Contemporânea de Contabilidade, v. 12, n. 27, p. 165-186, 2015.

\section{SECRETARIA DO TESOURO NACIONAL.}

Portaria STN n. 406, de 20 de junho de 2011. Aprova a $4^{\text {a }}$ Edição do Manual de Contabilidade Aplicado ao Setor Público (MCASP). Disponível em: http://www.tesouro.fazenda.gov.br/contabilidade . Acesso em: 13/05/2017.

Portaria STN n. 828, de 14 de

dezembro de 2011. Altera o prazo de implementação do Plano de Contas Aplicado ao Setor Público e dá outras providências.

Disponível em:

<http://www.editoramagister.com/doc_22715430 _PORTARIA_N_828_DE_14_DE_DEZEMBRO _DE_2011.aspx>.Acesso em 28/11/2017.

Portaria STN n. 231, de 29 de março de 2012. Altera o prazo de divulgação do cronograma de ações para adequação aos procedimentos contábeis apresentados na Portaria STN nº 828/2011 e dá outras providências. Disponível em: <http://sisweb.tesouro.gov.br/apex/f?p=2501:1:1 5559171595615::NO:::>.Acesso em 28/05/2017.

\section{SEVERINO, A. J. Metodologia do trabalho} científico. São Paulo: Cortez, 2016.

SLOMSKI V. et al. A demonstração do resultado econômico e sistemas de custeamento como instrumentos de evidenciação do cumprimento do princípio constitucional da eficiência, produção de governança e accountability no setor público: uma aplicação na ProcuradoriaGeral do Município de São Paulo. Revista de Administração Pública, v. 44, n. 4, p. 933-957, 2010.

Bianca dos Santos Costa
Mestre em Administração pela Universidade do
Estado de Santa Catarina (UDESC).

Estado de Santa Catarina (UDESC).
Jaqueline Gonçalves

Mestranda em Administração pela Universidade do Estado de Santa Catarina (UDESC).

Fabiano Maury Raupp

Doutor em Administração pela Universidade Federal da Bahia (UFBA). 